\title{
On Defining Irreducibility
}

\author{
Pascal Koiran \\ Laboratoire de l'Informatique du Parallélisme \\ Ecole Normale Supérieure de Lyon - CNRS \\ 46, allée d'Italie \\ 69364 Lyon Cedex 07, France \\ Pascal.Koiran@ens-lyon.fr
}

July 14, 2021

In this note we prove the following result.

Theorem 1 For any $n \geq 2$, irreducibility is not a definable property of real algebraic varieties of $\mathbb{R}^{n}$.

This means that for all $n \geq 2$, there exists no first-order sentence $F_{n}$ in the language $\mathcal{L}_{n}=\left(+,-, \times,=, 0,1, I_{n}\right)$ satisfying the following property: for any real algebraic variety $V$ of $\mathbb{R}^{n}$, if we interpret the $n$-ary predicate $I_{n}$ by membership to $V$ then $\mathbb{R} \models F_{n}$ if and only if $V$ is irreducible. Our proof will imply that irreducibility remains undefinable even if we add the exponential function to $\mathcal{L}_{n}$. It was inspired by Wilkie's proof that $C^{\infty}$ functions are not definable [8].

Conjecture 1 For any $n \geq 2$, irreducibility is not a definable property of complex algebraic varieties of $\mathbb{C}^{n}$.

It is not clear how this conjecture should be attacked. In particular, if one wishes to follow the same proof strategy as in this note, it is not clear by what structure the real exponential field should be replaced.

A study of definability in algebraically closed fields was initiated in [6]: in that paper we gave some examples of definable properties (for instance, dimension is definable), showed that connectivity of algebraic varieties is not definable, and conjectured that algebraic varieties are not definable among constructible sets. There is already a fairly large body of work on definability in o-minimal and stable structures, see for instance [1, 2, 3, 4, 5, 7]. 
Let $V_{n}$ be the real variety $\left\{(x, y) \in \mathbb{R}^{2} ; P_{n}(x, y)=0\right\}$ where

$$
P_{n}(x, y)=x^{2 n}-\left(1+y^{2}\right)^{2} .
$$

Note that $P_{n}=Q_{n} R_{n}$ with $Q_{n}(x, y)=x^{n}-1-y^{2}$ and $R_{n}(x, y)=x^{n}+$ $1+y^{2}$. Note also that there is a formula $F(n, x, y)$ of the language $\mathcal{L}_{\exp }=$ $\{+,-, \times,=$, exp, 0,1$\}$ such that whenever $n$ is a positive integer, $F(n, .,$. defines $V_{n}$ : take for $F$ the formula

$$
\exists z\left[e^{z}=x^{2} \wedge e^{n z}=\left(1+y^{2}\right)^{2}\right] .
$$

Here is another useful remark.

Lemma 1 For any integer $n \geq 0$, the real variety $V_{\mathbb{R}}\left(Q_{n}\right)$ defined by $Q_{n}$ is irreducible.

Proof. For $n=0, V_{\mathbb{R}}\left(Q_{n}\right)$ is the line $\{y=0\}$ and is therefore irreducible. For $n \geq 1, V_{\mathbb{R}}\left(Q_{n}\right)$ also has dimension 1 and it is not hard to check that $Q_{n}$ is irreducible over $\mathbb{C}$. It therefore follows from Lemma 2 below that $V_{\mathbb{R}}\left(Q_{n}\right)$ is irreducible.

Lemma 2 Let $Q \in \mathbb{R}[x, y]$ be a polynomial defining a real variety $V_{\mathbb{R}}(Q)$ of dimension 1. If $Q$ is irreducible over $\mathbb{C}$ then $V_{\mathbb{R}}(Q)$ is irreducible.

Proof. Let $V_{1}$ and $V_{2}$ be two real varieties such that $V_{\mathbb{R}}(Q)=V_{1} \cup V_{2}$. We shall see that $V_{1}=V_{\mathbb{R}}(Q)$ or $V_{2}=V_{\mathbb{R}}(Q)$. At least one of the $V_{i}$ must be of dimension 1. Assume for instance that this is true of $V_{1}$, and let $P_{1} \in \mathbb{R}[x, y]$ be a polynomial such that $V_{1}=V_{\mathbb{R}}\left(P_{1}\right)$. Since $V_{\mathbb{R}}\left(P_{1}\right) \cap V_{\mathbb{R}}(Q)$ has dimension 1 , the complex variety $V_{\mathbb{C}}\left(P_{1}\right) \cap V_{\mathbb{C}}(Q)$ also has dimension 1 . By irreducibility of $Q, V_{\mathbb{C}}(Q)$ is irreducible. It follows that $V_{\mathbb{C}}(Q) \subseteq V_{\mathbb{C}}\left(P_{1}\right)$ and therefore $V_{\mathbb{R}}(Q) \subseteq V_{\mathbb{R}}\left(P_{1}\right)$, i.e., $V_{\mathbb{R}}\left(P_{1}\right)=V_{\mathbb{R}}(Q)$.

Proposition $1 V_{n}$ is irreducible if and only if $n$ is even.

Proof. If $n$ is an odd number, the real varieties defined by $Q_{n}$ and $R_{n}$ are distinct (they are in fact disjoint). Hence $V_{n}$ is not irreducible in this case.

If $n$ is even, $R_{n}$ has no real zeros. $V_{n}$ is therefore irreducible by Lemma 1 . 
Proof of Theorem 11. We shall prove that irreducibility is not definable in dimension 2. The result for higher dimensions follows immediately from this special case.

Assume by contradiction that there exists a formula $F_{2}$ of $\mathcal{L}_{2}$ such that for any real variety $V$ of $\mathbb{R}^{2}$, if we interpret $I_{2}$ by membership to $V$ then $\mathbb{R} \models F_{2}$ if and only if $V$ is irreducible. Let $G(n)$ be the formula obtained from $F_{2}$ by replacing each instance $I_{2}(x, y)$ of $I_{2}$ in $F_{2}$ by $F(n, x, y)$. By Proposition 1, $G(n)$ is true whenever $n$ is an even integer, and is false whenever $G(n)$ is an odd integer. This is in contradiction with the o-minimality of the real exponential field (see 9] and the references there).

\section{References}

[1] J. T. Baldwin and M. Benedikt. Embedded finite models, stability theory, and the impact of order. In Proc. 13th IEEE Symposium on Logic in Computer Science, pages 490-500, 1998.

[2] O. V. Belegradek, A. Stolboushkin, and M. A. Taitslin. Extended ordergeneric queries. Annals of Pure and Applied Logic, 97:85-125, 1999.

[3] M. Benedikt, G. Dong, L. Libkin, and L. Wong. Relational expressive power of constraint query languages. Journal of the ACM, pages 1-34, 1998.

[4] M. Benedikt and L. Libkin. On the structure of queries in constraint query languages. In Proc. 11th IEEE Symposium on Logic in Computer Science, pages 25-34, 1996.

[5] M. Benedikt and L. Libkin. Languages for relational databases over interpreted structures. In Proc. 16th ACM Symposium on Principles of Database Systems, pages 87-98, 1997.

[6] O. Chapuis and P. Koiran. Definability of geometric properties in algebraically closed fields. LIP Research Report 98-32, Ecole Normale Suprieure de Lyon, 1998. To appear in Mathematical Logic Quarterly.

[7] S. Grumbach and J. Su. Queries with arithmetical constraints. Theoretical Computer Science, 173:151-181, 1997.

[8] A. J. Wilkie. On defining $C^{\infty}$. Journal of Symbolic Logic, 59(1):344, 1994. 
[9] A. J. Wilkie. O-minimality. Documenta Mathematica, I:633-636, 1998. http://www.mathematik.uni-bielefeld.de/documenta, Extra Volume ICM 1998. 